\section{Prevalence of swine Hepatitis E virus in Europe and its role as emerging zoonosis and food-borne pathogen}

\author{
Eleonora Sarno, ${ }^{1,2}$ Adriano ML Santoro, ${ }^{1}$ \\ Nicola Costanzo 3 \\ 'Dipartimento di Scienze Zootecniche e \\ Ispezione degli Alimenti di Origine \\ Animale, Università degli Studi di Napoli \\ Federico II, Italy; 'Institut für \\ Lebensmittelsicherheit und hygiene der \\ Universität Zürich, Zürich, Switzerland; \\ ${ }^{3}$ Dipartimento di Scienze della Salute, \\ Università Magna Græcia di Catanzaro, \\ Italy
}

\section{Abstract}

Hepatitis E virus (HEV) is responsible for enterically-transmitted acute hepatitis, a selflimiting disease in humans occurring via fecal-oral route. Hepatitis $\mathrm{E}$ virus is classified in the Hepeviridae family and consists of at least four genotypes. Genotypes 1 and 2 are restricted to humans and associated with epidemics in developing countries, whereas genotypes 3 and 4 are responsible for sporadic cases worldwide. Among all hepatitis (A, B, C and D), HEV is the only one with animal reservoirs. Swine HEV is widespread among pig population and several studies show phylogenetic relatedness between human and swine strains supporting the zoonotic hypothesis. Sporadic cases have also been linked to consumption of raw or undercooked food from infected animals. Therefore, swine strains of HEV pose not only a zoonotic risk but also food safety concerns. To assess this hypothesis, further investigations are required. The aim of the present article is to present a short overview on the HEV knowledge with respect to pig and human prevalence and its role as emerging zoonosis.

\section{Introduzione}

Il virus dell'epatite E (HEV) è l'agente eziologico responsabile nell'uomo di una forma acuta e auto-limitante di epatite non-A e nonB con sintomatologia gastroenterica, non soggetta a cronicizzazione. Nella maggior parte dei casi la forma clinica è di lieve entità con un tasso di mortalità dell'1-4\% che può però raggiungere il $20 \%$ in donne in gravidanza (Purcell e Emerson, 2008) ed essere letale per soggetti immunocompromessi.

La trasmissione avviene per via oro-fecale.
Nei Paesi in via di sviluppo l'infezione è endemica e rappresenta un importante problema di sanità pubblica. In queste aree, infatti, si manifesta con focolai epidemici e la trasmissione è dovuta alla contaminazione delle fonti d'acqua.

Fino a qualche anno fa, i Paesi industrializzati erano considerati indenni e i casi di malattia erano registrati in soggetti al ritorno da viaggi in zone endemiche. Attualmente nelle aree sviluppate dove l'infezione ha carattere sporadico, si registrano sempre più di frequente casi di epatite $\mathrm{E}$ autoctona contratta a seguito del contatto con animali infetti (categorie professionali a rischio come veterinari e allevatori) (Meng et al., 2002) oppure acquisita per via alimentare.

Di tutti i virus noti per essere causa di epatite (A, B, C e D) HEV è l'unico a riconoscere nella specie suina (e in altri animali) il principale reservoir in natura. Nel 1997 fu per la prima volta isolato e caratterizzato negli USA un ceppo animale proveniente dal suino denominato swine HEV (Meng et al., 1997). Successivamente, la presenza di anticorpi anti-HEV è stata documentata in altre specie animali: cinghiale in primo luogo, pollo, coniglio, ratto, cervo, bovino e ovino (Sonoda et al., 2004; Payne et al., 1999; Zhao et al., 2009; Johne et al., 2010; Tei et al., 2003; Hu e Ma, 2010; Wang e Ma, 2010).

Nel suino la patologia ha un decorso subclinico e asintomatico. L'infezione avviene generalmente a 6-8 settimane di età e coincide con il declino dell'immunità passiva acquisita attraverso il colostro. La sieroconversione avviene tra le 14 e le 17 settimane di vita corrispondenti al picco di viremia. Il picco di immunoglobuline G (Ig G) anti-HEV si registra intorno alla ventiduesima settimana (de Deus et al., 2008) quando le immunoglobuline M (Ig M) iniziano a diminuire.

Dal punto di vista tassonomico inizialmente fu identificato come Calicivirus, quindi come Togavirus. Rivisitata questa classificazione a tutt'oggi HEV è il solo membro del genere Hepevirus, famiglia Hepeviridae (Emerson et al., 2004). Il virus presenta un capside a simmetria icosaedrica ed è privo di envelope. Il genoma virale è un singolo filamento positivo di RNA con dimensioni di 7,2 kb. Include due brevi regioni non codificanti che circondano la regione codificante che a sua volta contiene tre open reading frames (ORFs). Le regioni ORF1, ORF2 e ORF3 codificano rispettivamente per una proteina non strutturale, per la proteina strutturale del capside responsabile della risposta anticorpale e candidata come possibile vaccino (Emerson e Purcell, 2003), e per una piccola proteina la cui funzione non è ancora ben chiara ma che sembra coadiuvare l'attività della proteina codificata dalla regione ORF2 (Okamoto, 2007).

Benché sia noto un solo sierotipo (Emerson
Correspondence: Eleonora Sarno, Dipartimento di Scienze Zootecniche e Ispezione degli Alimenti di Origine Animale, Università degli Studi di Napoli Federico II, Napoli, Italy. Tel. +39.081.2536054 - Fax: +39.081.458683.

E-mail: eleonora.sarno@unina.it

Key words: Swine hepatitis E virus, Emerging zoonosis, Food-borne pathogen.

Conflict of interests: the authors declare no potential conflict of interests.

Received for publication: 15 January 2013.

Revision received: 19 March 2013.

Accepted for publication: 19 March 2013.

This work is licensed under a Creative Commons Attribution 3.0 License (by-nc 3.0).

(C) Copyright E. Sarno et al., 2013

Licensee PAGEPress, Italy

Italian Journal of Food Safety 2013; 2:e13

doi:10.4081/ijfs.2013.e13

e Purcell, 2003), sono attualmente riconosciuti quattro genotipi nei mammiferi, con differente distribuzione geografica e di ospite (Lu et al., 2006), più uno aviario il cui genoma mostra il 48-60\% di omologia nucleotidica con ceppi umani e la sua collocazione non è ben chiara (Haqshenas et al., 2001). I genotipi $1 \mathrm{e}$ 2 sono isolati nell'uomo e distribuiti in Asia, Africa e Messico, rispettivamente. Il genotipo 3 è quello circolante in Europa e USA mentre il 4 ha per lo più distribuzione asiatica. Entrambi sono isolati nell'uomo e nelle diverse specie animali.

Oggigiorno la natura ubiquitaria del virus nella popolazione suina è testimoniata dai numerosi studi di sieroprevalenza (Ig G antiHEV), dagli studi di omologia filogenetica tra i ceppi suini e umani e dai casi clinici segnalati di epatite E autoctona nell'uomo. Tali evidenze sollevano sempre più preoccupazione per la salute pubblica in termini di rischio zoonotico e di sicurezza alimentare (Lewis et al., 2010; Meng, 2010).

Lo scopo di tale rassegna è di fornire una breve sintesi sulle attuali conoscenze scientifiche del virus, con particolare riferimento alla prevalenza nella popolazione suina in ambito europeo, e di inquadrare tale patologia, cosiddetta emergente, come zoonosi e malattia a trasmissione alimentare (MTA).

\section{Prevalenza del virus dell'epatite E}

A causa della mancanza di metodi standard per 
l'isolamento del virus da campioni e da matrici alimentari che consentano un'adeguata comparazione dei dati sperimentali presenti in letteratura e, considerando che la coltura cellulare in vitro sembra essere poco efficiente e richiedere elevati titoli anticorpali, una stima precisa della prevalenza di HEV non è di semplice esecuzione. Tuttavia, kit immunoenzimatici come l'enzyme-linked immunosorbent assay (ELISA) per l'identificazione diretta degli anticorpi anti-HEV sono disponibili in commercio e, unitamente a tecniche di biologia molecolare come la reverse transcriptionpolymerase chain reaction (RT-PCR), trovano ampio impiego per scopi diagnostici.

In Italia si stima che la sieroprevalenza nella popolazione umana, che attesta l'avvenuto contatto con il virus attraverso la produzione di anticorpi, varia secondo la regione geografica ma si mantiene su valori concordanti: $3,3 \%$ al Nord (Romanò et al., 2011), 2,9\% al Centro (Vulcano et al., 2007), 3,8\% al Sud (Cacciola et al., 2011), e 4,3\% nelle isole (Sardegna) (Masia et al., 2009). Non molto distante è il valore del $4,9 \%$ riportato in Svizzera (Kaufmann et al., 2011).

Una prevalenza maggiore in soggetti appartenenti alle categorie professionali a rischio, come veterinari e allevatori suini, è stata osservata da Vulcano et al. (2007) con un valore indicato del $3,3 \%$ e da Galiana et al. (2008) con un valore indicato del $19 \%$.

Nella Tabella 1 sono indicati gli studi di prevalenza (2009-2012) nel suino domestico e nei prodotti da esso derivati. Da quanto emerge e tenendo conto che sono stati presi in considerazione studi condotti negli ultimi tre anni, la prevalenza del virus ottenuta da campioni di succo muscolare varia dal $49 \%$ del centro Europa (Wacheck et al., 2012) al 64\% del sudovest Europa (Casas et al., 2011). Una prevalenza del $31 \%$ è stata evidenziata in Francia (Rose et al., 2011), mentre in Italia uno studio condotto su campioni di siero suino da Di Bartolo et al. (2011) ha mostrato una positività dell' $87 \%$. Gli studi di biologia molecolare a partire da campioni di bile rivelano dati contrastanti in Italia: mentre Masia et al. (2009) ha segnalato una prevalenza del 6,3\%, Di Bartolo et al. (2011) ha individuato una positività del $51,1 \%$. Similmente, in Belgio, indagini di biologia molecolare su campioni di feci evidenziano la presenza del virus nel 7\% dei casi (Hakze-Van Der Honing et al., 2011), e nel 49\% (Breum et al., 2010) in Danimarca. I campioni di fegato risultati positivi in Germania e Francia si sono attestati al 4\% (Wenzel et al., 2011; Rose et al., 2011), mentre in Ungheria al 31\% (Forgách et al., 2010).

Negli ultimi anni si è inoltre registrato un aumento del numero di casi clinici nell'uomo. In Gran Bretagna il virus è stato isolato da una donna in corso di epatite acuta che riferiva di aver ingerito salsicce poco cotte e non avere viaggiato in aree endemiche. Il virus mostrava piena omologia con quello isolato e circolante nella popolazione suina sullo stesso territorio (Banks et al., 2004). In Portogallo un paziente con infezione acuta che riferiva di non avere viaggiato in aree endemiche e di non aver avuto contatto con animali ma piuttosto di aver ingerito salsicce poco cotte, in corso di diagnosi differenziale, risultava positivo per Ig M e Ig $\mathrm{G}$ anti-HEV mediante test ELISA (Duque et al., 2012). Il primo caso di epatite autoctona in Francia è riportato da Tessè et al. (2012) in una giovane donna immunocompromessa. Nonostante la paziente riferisse di non avere viaggiato in zone endemiche e di non aver avuto contatto con animali né di aver consumato cibo poco cotto, veniva isolato il genotipo 4 che normalmente è circolante in Asia e non in Europa. Casi autoctoni di epatite da HEV sono stati descritti anche in Germania (Veitt $e t$ al., 2011) e Italia (Zanetti et al., 1999).

\section{Discussione}

I virus a trasmissione alimentare rappresentano la seconda causa principale di focolai di tossinfezione nell'Unione Europea (UE) dopo Salmonella spp. (EFSA, 2011). Nel 2009 essi hanno provocato il $19 \%$ di tutti i focolai sviluppatisi in UE (FAO/WHO, 2008). Ciononostante, le informazioni e le conoscenze del virus dell'epatite $\mathrm{E}$ e la diffusione dell'infezione nella popolazione umana e in quella suina sono ancora relativamente insufficienti. Tuttavia, l'ipotesi che l'infezione da HEV possa rappresentare una zoonosi emergente nei Paesi industrializzati nasce dalle numerose osservazioni riportate dalla letteratura recente.

In primo luogo importanti evidenze che supportano la possibilità di trasmissione zoonotica provengono dagli studi di correlazione filo-

Tabella 1. Studi di prevalenza in Europa (2008-2012) del virus dell'epatite E mediante test immunoenzimatici (enzyme-linked immunosorbent assay) e metodiche molecolari (real time polymerase chain reaction) nel suino e prodotti da esso derivati.

\begin{tabular}{|c|c|c|c|}
\hline Referenza & Paese & $\begin{array}{l}\text { Test di laboratorio } \\
\text { (tipo di campione) }\end{array}$ & Prevalenza (\%) \\
\hline Wacheck et al., 2012 & Svizzera & ELISA (succo muscolare) & 49 \\
\hline Casas et al., 2011 & Spagna & ELISA (succo muscolare) & 64 \\
\hline Casas et al., 2011 & Spagna & ELISA (siero) & 64 \\
\hline Di Bartolo et al., 2011 & Italia & ELISA (siero) & 87 \\
\hline Rose et al., 2011 & Francia & ELISA (siero) & 31 \\
\hline Breum et al., 2010 & Danimarca & ELISA (siero) & 73,2 \\
\hline Baechlein et al., 2010 & Germania & ELISA (siero) & 49,8 \\
\hline Martinelli et al., 2011 & Italia & ELISA (siero) & 50,2 \\
\hline Di Bartolo et al., 2011 & Italia & RT-PCR (bile) & 51,1 \\
\hline Masia et al., 2009 & Italia & RT-PCR (bile) & 6,3 \\
\hline Kosinova et al., 2012 & Repubblica Ceca & RT-PCR (bile) & 34,9 \\
\hline Kosinova et al., 2012 & Repubblica Ceca & RT-PCR (feci) & 22,2 \\
\hline Hakze-Van Der Honing et al., 2011 & Olanda & RT-PCR (feci) & 15 \\
\hline Hakze Van Der Honing et al., 2011 & Belgio & RT-PCR (feci) & 7 \\
\hline McCreary et al., 2008 & Gran Bretagna & RT-PCR (feci) & 21,5 \\
\hline Steyer et al., 2011 & Slovenia & RT-PCR (feci) & 20,3 \\
\hline Widèn et al., 2011 & Svezia & RT-PCR (feci) & 29,6 \\
\hline Breum et al., 2010 & Danimarca & RT-PCR (feci) & 49,5 \\
\hline Di Martino et al., 2010 & Italia & RT-PCR (feci) & 7,3 \\
\hline Berto et al., 2012 & Portogallo & RT-PCR (feci) & 22 \\
\hline Di Bartolo et al., 2011 & Italia & RT-PCR (feci) & 33,3 \\
\hline Forgách et al., 2010 & Ungheria & RT-PCR (feci) & 21 \\
\hline Di Bartolo et al., 2011 & Italia & RT-PCR (fegato) & 20,8 \\
\hline Forgách et al., 2010 & Ungheria & RT-PCR (fegato) & 31 \\
\hline Wenzel et al., 2011 & Germania & RT-PCR (fegato) & 4 \\
\hline Kaba et al., 2010 & Francia & RT-PCR (fegato) & 2,5 \\
\hline Rose et al., 2011 & Francia & RT-PCR (fegato) & 4 \\
\hline
\end{tabular}

ELISA, enzyme-linked immunosorbent assay; RT-PCR, real time polymerase chain reaction. 
genetica tra ceppi umani e suini isolati sullo stesso territorio geografico e dalle consistenti analogie nucleotidiche e amminoacidiche esistenti tra di essi. Il ceppo umano isolato da Banks et al. (2004) mostrava ben il $100 \%$ d'identità amminoacidica con il ceppo suino circolante sullo stesso territorio. Ciò induce fortemente a pensare a un possibile passaggio da una specie a un'altra. Tuttavia, la capacità del virus di superare la barriera di specie uomo-animale non è stata ancora dimostrata. Quello che invece è stato dimostrato e confermato da infezioni sperimentali è la capacità di HEV di superare la barriera suino-primati non umani (Meng et al., 1998).

A confermare l'origine zoonotica della malattia vi è anche la più alta sieroprevalenza osservata nelle categorie esposte direttamente al contatto con animali infetti e/o utensili contaminati da feci (veterinari, allevatori, personale di allevamento, ecc.) rispetto alla popolazione generale.

Per quanto concerne l'ipotesi di MTA bisogna ricordare che le forme autoctone di epatite $\mathrm{E}$ citate dalla letteratura sono per lo più associate al consumo di alimenti di origine suina. Ciò è testimoniato dai vari casi clinici in cui HEV è stato isolato da pazienti senza anamnesi di viaggio in zone endemiche e che avevano riferito di aver ingerito alimenti poco cotti.

Perché l'ipotesi della trasmissione zoonotica sia definitivamente confermata sono ancora necessari, in campo veterinario, approfonditi studi circa i meccanismi e i fattori di rischio coinvolti nell'infezione per via alimentare, così come è necessario un miglioramento dei test diagnostici per l'isolamento e l'identificazione del virus stesso (Wilhelm et al., 2011).

Inoltre, la crescente prevalenza nella popolazione animale corrisponderebbe a un rischio maggiore di trasmissione all'uomo qualora tale ipotesi fosse valida.

\section{Conclusioni}

Considerando quanto detto finora, ulteriori studi sono necessari in previsione della valutazione e gestione del rischio per la salute pubblica. Secondo il gruppo di esperti scientifici sui pericoli biologici [panel on biological hazards (BIOHAZ)] dell'EFSA, misure efficaci volte a contenere la diffusione del virus dovrebbero incentrarsi sulla prevenzione della contaminazione a tutti i livelli della produzione, anziché sull'eliminazione 0 inattivazione dagli alimenti contaminati. Alimenti che tra l'altro non vanno in contro a modificazioni organolettiche visibili né a deterioramento poiché, a differenza dei batteri potenzialmente patogeni e responsabili di MTA, i virus sono parassiti endocellulari obbligati e necessitano della cellula ospite per la replicazione e non del substrato alimentare. Inoltre, essendo privo di envelope, HEV resiste bene a valori estremi di $\mathrm{pH}$ (alcalini e acidi), all'essicazione e persiste a lungo nell'ambiente esterno. Tuttavia, l'inattivazione è stata comunque dimostrata $\mathrm{a} 71^{\circ} \mathrm{C}$ per 20 min (Bernaud et al., 2012) per cui attualmente la cottura accurata a cuore del prodotto è l'unica misura efficace per eliminare 0 inattivare il virus dell'epatite E. La prevenzione deve basarsi inoltre sulla corretta informazione tecnico-scientifica e il gruppo di esperti BIOHAZ raccomanda di dissuadere le persone con malattie epatiche o deficienze immunitarie e le donne in stato di gravidanza dal consumare carne e fegato di maiale 0 cinghiale poco cotti.

\section{Bibliografia}

Baechlein C, Schielke A, Johne R, Ulrich RG, Baumgaertner W, Grummer B, 2010. Prevalence of Hepatitis E virus-specific antibodies in sera of German domestic pigs estimated by using different assays. Vet Microbiol 144;187-91.

Banks M, Bendall R, Grierson S, Heath G, Mitchell J, Dalton H, 2004. Human and porcine hepatitis E virus strains, United Kingdom. Emerg Infect Dis 5:953-5.

Barnaud E, Rogée S, Garry P, Rose N, Pavio N, 2012. Thermal inactivation of infectious hepatitis e virus in experimentally contaminated food. Appl Environ Microb 78:5153-9.

Berto A, Mesquita JR, Hakze-van der Honing R, Nascimento MS, van der Poel WH, 2012. Detection and characterization of Hepatitis E virus in domestic pigs of different ages in Portugal. Zoonoses and Public Health 59;477-81.

Breum Sø, Hjulsager CK, de Deus N, Segalés J, Larsen LE, 2010. Hepatitis E virus is highly prevalent in the Danish pig population. Vet Microbiol 146:144-9.

Cacciola I, Messineo F, Cacopardo B, Di Marco V, Galli C, 2011. Hepatitis E virus infection as a cause of acute hepatitis in Southern Italy. Digest Liver Dis 43:996-1000.

Casas M, Pina S, Peralta B, Mateu E, Casal J, Martín M, 2011. Comparison of muscle fluid and serum for detection of antibodies against hepatitis $\mathrm{E}$ virus in slaughter pigs. Vet J 190:179-80.

de Deus N, Casas M, Peralta B, Nofrarias M, Pina S, Martin M, Segales J, 2008. Hepatitis E virus infection dynamics and organic distribution in naturally infected pigs in a farrow-to-finish farm. Vet Microbiol 132:19-28.

Di Bartolo I, Ponterio E, Castellini L, Ostanello F, Ruggeri FM, 2011. Viral and antibody
HEV prevalence in swine at slaughterhouse in Italy. Vet Microbiol 149:330-8.

Di Martino B, Di Profio F, Martella V, Di Felice E, Di Francesco CE, Ceci C, Marsilio F, 2010. Detection of hepatitis $\mathrm{E}$ virus in slaughtered pigs in Italy. Arch Virol 155;103-6.

Duque V, Ventura C, Seixas D, da Cunha S, Meliço-Silvestre A, 2012. First report of acute autochthonous hepatitis $\mathrm{E}$ in Portugal. J Infect Dev Ctries 6:201-3.

EFSA, 2011. The European Union summary report on trends and sources of zoonoses, zoonotic agents and food-borne outbreaks in 2009. EFSA Journal 9:2090. Disponibile al sito: http://www.efsa.europa.eu/en/efsajournal/pub/2090.htm

Emerson SU, Anderson D, Arankalle VA, Meng XJ, Purdy M, Schlauder GG, Tsarev SA, 2004. Hepevirus. In: Fauquet CM, Mayo MA, Maniloff J, Desselberger U, Ball LA, eds. Virus taxonomy, 8th report of the ICTV. Elsevier/Academic Press, London, UK, pp 851-5.

Emerson SU, Purcell RH, 2003. Hepatitis E virus. Rev Med Virol 13:145-54.

FAO/WHO, 2008. Microbiological hazards in fresh leafy vegetables and herbs: meeting report. Food and Agriculture Organization of the United Nations/World Health Organization ed., Roma, Italy.

Forgách P, Nowotny N, Erdélyi K, Boncz A, Zentai J, Szucs G, Reuter G, Bakonyi T, 2010. Detection of hepatitis $\mathrm{E}$ virus in samples of animal origin collected in Hungary. Vet Microbiol 143:106-16.

Galiana C, Fernández-Barredo S, García A, Gómez MT, Pérez-Gracia MT, 2008. Short report: occupational exposure to hepatitis E virus (HEV) in swine workers. Am J Trop Med Hyg 78:1012-5.

Hakze-van der Honing RW, van Coillie E, Antonis AF, van der Poel WH, 2011. First isolation of hepatitis E virus genotype 4 in Europe through swine surveillance in The Netherlands and Belgium. PloS One 6:e22673.

Haqshenas G, Shivaprasad HL, Woolcock PR, Read DH, Meng XJ, 2001. Genetic identification and characterization of a novel virus related to human hepatitis $\mathrm{E}$ virus from chickens with hepatitis-splenomegaly syndrome in the United States. J Gen Virol 82:2449-62.

Hu GD, Ma X, 2010. [Detection and sequences analysis of bovine hepatitis E virus RNA in Xinjiang autonomous region]. [Articolo in cinese]. Bing Du Xue Bao/Chinese journal of virology 26:27-32.

Johne R, Plenge-Bönig A, Hess M, Ulrich RG, Reetz J, Schielke A, 2010. Detection of a novel hepatitis E-like virus in faeces of wild rats using a nested broad-spectrum RT-PCR. J Gen Virol 91:750-8. 
Kaba M, Davoust B, Marié JL, Colson P, 2010. Detection of hepatitis $\mathrm{E}$ virus in wild boar (Sus scrofa) livers. Vet J 186;259-61.

Kaufman A, Kenfak-Foguena A, André C, Canellini G, Bürgisser P, Moradpour D, Darling KEA, Cavassini M, 2011. Hepatitis E virus seroprevalence among blood donors in Southwest Switzerland. PLoS One 6:e21150.

Kosinova E, Bendova J, Vasickova P, Smitalova $\mathrm{R}$, Prodelalova J, 2012. The prevalence of hepatitis $\mathrm{E}$ virus in piglets on Czech pig production farms and phylogenetic analysis of recovered isolates. Vet Med-Czech $57 ; 115-20$.

Lewis HC, Wichmann 0, Duizer E, 2010. Transmission routes and risk factors for autochthonous hepatitis E virus infection in Europe: a systematic review. Epidemiol Infect 138:145-66.

Lu L, Li C, Hagedorn CH, 2006. Phylogenetic analysis of global hepatitis E virus sequences: genetic diversity, subtypes and zoonosis. Rev Med Virol 16:5-36.

Martinelli N, Luppi A, Cordioli P, Lombardi G, Lavazza A, 2011. Prevalence of hepatitis E virus antibodies in pigs in Northern Italy. Infect Ecol Epidemiol 1:7331.

Masia G, Orrù G, Liciardi M, Desogus G, Coppola RC, Murru V, Argiolas M, Orrù G, 2009. Evidence of hepatitis E virus (HEV) infection in human and pigs in Sardinia, Italy. J Prev Med Hyg 50:227-31.

McCreary C, Martelli F, Grierson S, Ostanello F, Nevel A, Banks M, 2008. Excretion of hepatitis $\mathrm{E}$ virus by pigs of different ages and its presence in slurry stores in the United Kingdom. Vet Rec 163;261-5.

Meng XJ, 2010. Hepatitis E virus: animal reservoirs and zoonotic risk. Vet Microbiol 140:256-65.

Meng XJ, Halbur PG, Shapiro MS, Govindarajan S, Bruna JD, Mushahwar IK, Purcell RH, Emerson SU, 1998. Genetic and experimental evidence for crossspecies infection by swine hepatitis $\mathrm{E}$ virus. $\mathrm{J}$ Virol 72:9714-21.

Meng XJ, Purcell RH, Halbur PG, Lehman JR, Webb DM, Tsareva TS, Haynes JS, Thacker BJ, Emerson SU, 1997. A novel virus in swine is closely related to the human hepatitis E virus. Proc Natl Acad Sci USA 94:9860-5.

Meng XJ, Wiseman B, Elvinger F, Guenette DK, Toth TE, Engle RE, Emerson SU, Purcell RH, 2002. Prevalence of antibodies to hepatitis E virus in veterinarians working with swine and in normal blood donors in the United States and other countries. J Clin Microbiol 40:117-22.

Okamoto H, 2007. Genetic variability and evolution of hepatitis $\mathrm{E}$ virus. Virus Res 127:216-28.

Payne CJ, Ellis TM, Plant SL, Gregory AR, Wilcox GE, 1999. Sequence data suggests big liver and spleen disease virus (BLSV) is genetically related to hepatitis $\mathrm{E}$ virus. Vet Microbiol 68:119-25.

Purcell RH, Emerson SU, 2008. Hepatitis E: an emerging awareness of an old disease. J Hepatol 48:494-503.

Romanò L, Paladini S, Tagliacarne C, Canuti M, Bianchi S, Zanetti AR, 2011. Hepatitis E in Italy: a long-term prospective study. J Hepatol 54:34-40.

Rose N, Lunazzi A, Dorenlor V, Merbah T, Eono F, Eloit M, Madec F, Pavio N, 2011. High prevalence of Hepatitis E virus in French domestic pigs. Comp Immunol Microb 34:419-27.

Sonoda H, Abe M, Sugimoto T, Sato Y, Bando M, Fukui E, Mizuo H, Takahashi M, Nishizawa T, Okamoto H, 2004. Prevalence of hepatitis E virus (HEV) Infection in wild boars and deer and genetic identification of a genotype 3 HEV from a boar in Japan. $\mathrm{J}$ Clin Microbiol 42:5371-4.

Steyer A, Naglic T, Mocilnik T, Poljšak-Prijatelj M, Poljak M, 2011. Hepatitis E virus in domestic pigs and surface waters in Slovenia: prevalence and molecular characterization of a novel genotype 3 lineage. Infect Genet Evol 11;1732-7.

Tei S, Kitajima N, Takahashi K, Mishiro S, 2003. Zoonotic transmission of hepatitis $\mathrm{E}$ virus from deer to human beings. Lancet 362:371-3.

Tessé S, Lioure B, Fornecker L, Wendlingc MJ, Stoll-Keller F, Bigaillon C, Nicanda E, 2012. Circulation of genotype 4 hepatitis E virus in Europe: first autochthonous hepatitis E infection in France. J Clin Virol 54:197-
200.

Veitt R, Reichardt M, Wenzel J, Jilg W, 2011. [Autochthone Hepatitis-E-Virus-Infektion als Ursache der akuten Hepatitis in Deutschland - eine Kasuistik]. [Articolo in tedesco]. Z Gastroenterol 49:42-6.

Vulcano A, Angelucci M, Candelora E, Martini V, Patti AM, Mancini C, Santi AL, Calvari A, Casagni L, Lamberti A, 2007. HEV prevalence in the general population and among workers at zoonotic risk in Latium Region. Ann Ig 19:181-6.

Wacheck S, Sarno E, Märtlbauer E, Zweifel C, Stephan R, 2012. Seroprevalence of antiHEV and anti-Salmonella antibodies in pigs at slaughter in Switzerland. J Food Protect 75:1483-5.

Wang Y, Ma X, 2010. [Detection and sequences analysis of sheep hepatitis E virus RNA in Xinjiang autonomous region]. [Articolo in cinese]. Wei Sheng Wu Xue Bao/Acta microbiologica Sinica 50:937-41.

Wenzel JJ, Preiss J, Schemmerer M, Huber B, Plentz A, Jilg W, 2011. Detection of hepatitis $\mathrm{E}$ virus (HEV) from porcine livers in Southeastern Germany and high sequence homology to human HEV isolates. J Clin Virol 52:50-4.

Widèn F, Sundqvist L, Matyi-Toth A, Metreveli G, Belàk S, Hallgren G, Norder H, 2011. Molecular epidemiology of hepatitis E virus in humans, pigs and wild boars in Sweden. Epidemiol Infect 139;361-71.

Wilhelm BJ, Rajic A, Greig J, Waddell L, Trottier G, Houde A, Harris J, Borden LN, Price C, 2011. A systematic review/meta-analysis of primary research investigating swine, pork or pork products as a source of zoonotic hepatitis E virus. Epidemiol Infect 139:1127-44.

Zanetti AR, Schlauder GG, Romano L, Tanzi E, Fabris P, Dawson GJ, Mushahwar IK, 1999. Identification of a novel variant of hepatitis E virus in Italy. J Med Virol 57:356-60.

Zhao C, Ma Z, Harrison TJ, Feng R, Zhang C, Qiao Z, Fan J, Ma H, Li M, Song A, Wang Y, 2009. A novel genotype of hepatitis $E$ virus prevalent among farmed rabbits in China. J Med Virol 81:1371-9. 